evidence for AI (18), their usual practice (11), unlicensed regime (7), and safety concerns (4). 100\% of the respondents who favoured colectomy cited safety concerns as their main reason for deciding against continuing medical therapy.

\begin{tabular}{ll} 
Abstract PTH-117 Table 1 & N (\%) \\
\hline & $41(20.1)$ \\
\hline Job Role & $144(70.6)$ \\
IBD specialist & $21(10.3)$ \\
Gastroenterologist with interest in IBD & \\
Gastroenterologist with main interest in other areas & $82(39.6)$ \\
Type of institution & $126(60.9)$ \\
University teaching hospital & \\
District General hospital & \\
ASUC patients (per year) & $15(7.2)$ \\
$<5$ & $83(39.9)$ \\
$5-9$ & $80(38.5)$ \\
$10-19$ & $30(14.4)$ \\
$>20$ & \\
Colectomy as alternative to IVCS & $32(15.4)$ \\
Yes & $176(84.6)$ \\
No & \\
Provision of Lap colectomy in ASUC & $81(38.9)$ \\
Yes & $86(41.3)$ \\
No & $16(7.7)$ \\
Unsure & $25(12)$ \\
Proposionally & \\
\hline $25 \%-49 \%$ & $22(10.6)$ \\
\hline $50-74 \%$ & $33(15.9)$ \\
\hline
\end{tabular}

Conclusions There is significant variation in practice in the use of infliximab rescue therapy in ASUC. There is an urgent need for development of care pathways to standardise practice.

\section{PTH-118 MUCOSAL TISSUE SHORT CHAIN FATTY ACIDS CONTRIBUTE TO PREDICTION OF POUCHITIS IN RESTORATIVE PROCTOCOLECTOMY}

\footnotetext{
1,2Jonathan Segal ${ }^{*},{ }^{2}$ Magali Sarafian, ${ }^{2}$ Alexandros Pechlivanis, ${ }^{2}$ Ivan Jose Serrano Contreras, ${ }^{2}$ Jerusa Brignardello, ${ }^{2}$ Yih-harn Siaw, 1,2 Lucia Braz, ${ }^{1,2}$ Susan Clark, 1,2Elaine Holmes, ${ }^{1,2}$ Ailsa Hart. ${ }^{1}$ St Marks Hospital, Middlesex, UK; ${ }^{2}$ Imperial College London, London, UK
}

\subsection{6/gutjnl-2019-BSGAbstracts.177}

Background Restorative proctocolectomy is a surgical option in patients with ulcerative colitis who become refractory to medical therapy. Short chain fatty acids (SCFA) are organic fatty acids with 1-6 carbons which arise from bacterial metabolism from carbohydrates entering the colon. Various studies have implicated SCFA in both the development of IBD and flares of IBD. Furthermore, it has been shown that SCFA concentrations are significantly lower in faecal samples from patients with pouchitis when compared with healthy controls.

Our study aimed to assess longitudinal changes in SCFA that occur in a pouch to determine if they can predict or are associated with the development of pouchitis. To date no study has analysed short chain fatty acids in mucosal biopsy tissue from these patients.

Methods Patients who underwent restorative proctocolectomy at a single centre underwent pouchoscopy at the time of restoration of continuity and then every 6 months for a year. Biopsies from the pouch were retrieved from the pouch body. Pouchitis was defined using the pouch disease activity index. The development of pouchitis was assessed at months 6 and 12 months.

Biopsies samples were snap frozen at time of biopsy and stored in $-80^{\circ} \mathrm{C}$. Samples were thawed and weighed. Sterile water and Methyl tertiary-butyl ether with internal standard (IS) were added with a ratio of $20 \mathrm{mg}$ of sample:50 $\mu \mathrm{L}$ of $\mathrm{H} 20: 250 \mu \mathrm{L}$ of $\mathrm{MTBE}$ and IS with a further $4 \mu \mathrm{L}$ of hydrochloric acid added to each sample. $30 \mu \mathrm{L}$ of the polar phase was then placed into silanized Eppendorf tubes. $150 \mu \mathrm{L}$ of derivatiser was added to each sample and the cap of the tube applied immediately. These were then incubated for 45 minutes at $60^{\circ} \mathrm{C}$ in an oven. $70 \mu \mathrm{L}$ from the silanised vial was placed into vial inserts and analysed in the gas chromatography mass spectrometry machine. (GC-MS). SCFA were measured using an Agilent 7000C Triple Quadrupole GC/MS-MS System according to a previously published method. Simca was used for multivariate analysis and T-tests were used for univariate analysis.

Results There were 56 biopsy samples. There were 22 patients (17 males); 16 UC and 6 FAP patients with longitudinal follow up. The median age of the cohort was 40 years (range 20-60 years). Of the UC patients four developed pouchitis within one year.

When comparing UC patients at the time of closure of ileostomy, there were there were significant decreases in caproic acid $(4674 \mu \mathrm{M}$ vs $12217 \mu \mathrm{M} \quad \mathrm{p}<0.01)$, valeric acid $(1580 \mu \mathrm{M}$ vs $3695 \mu \mathrm{M} \mathrm{p}=0.01)$, isovolaric acid $(721 \mu \mathrm{M}$ vs $2940 \mu \mathrm{M} p=0.05)$, isobutyric acid $35072 \mu \mathrm{M}$ vs $76074 \mu \mathrm{M}$ $\mathrm{p}=0.03)$ and lactic acid $(1580 \mu \mathrm{M}$ vs $3732 \mu \mathrm{M} \quad \mathrm{p}=0.02)$ between those who developed pouchitis within a year and those who did not develop pouchitis at 1 year.There were no significant differences detected between UC patients and FAP patients at each time point analysis.

Conclusion The study has suggested that a decrease in SCFA found in the mucosal tissue at time of closure of ileostomy may predict onset of pouchitis within a year. This study is the first to demonstrate that SCFA can be analysed from biopsies. Future studies need to determine factors that may contribute to tissue SCFA levels which may help develop a potential therapeutic target to optimise and potentially reduce the incidence of pouchitis.

\section{PTH-119 HOW ACCEPTABLE IS A 'TREAT TO TARGET' (T2T) APPROACH TO IBD PATIENTS IN CLINICAL REMISSION?}

Jenelyn Carbonell, John Kane, Omer Mandour, Alvin Odouri O'Chieng, Matt Pinder, Rebecca McKay, John Hamlin, Christian Selinger*. Leeds Teaching Hospitals NHS Trust, Leeds, UK

\subsection{6/gutjnl-2019-BSGAbstracts.178}

Background Treatment algorithms for IBD are shifting from traditional symptom based pathways to a 'treat to target' T2T approach aiming for clinical remission and absence of mucosal inflammation. We aimed to establish whether patients with IBD in clinical remission agree to this more intense approach. 